Article

\title{
Size Control of Carbon Encapsulated Iron Nanoparticles by Arc Discharge Plasma Method
}

\author{
Mohammad Reza Sanaee ${ }^{1,2, *}$, Stefanos Chaitoglou ${ }^{1}$, Noemí Aguiló-Aguayo ${ }^{1,3}$ and \\ Enric Bertran ${ }^{1, *}$ \\ 1 FEMAN Group Department of Applied Physics, Institute of Nanoscience and Nanotechnology, \\ Universitat de Barcelona, Martíi Franquès, 1, Barcelona E08028, Spain; schaitog@gmail.com (S.C.); \\ noeaguilo@gmail.com (N.A.-A.) \\ 2 Department of Nano Chemical Engineering, Faculty of Advanced Technologies, Shiraz University, \\ Shiraz 71946, Iran \\ 3 Research Institute of Textile Chemistry and Textile Physics, University of Innsbruck, Hoechsterstrasse 73, \\ Dornbirn 6850, Austria \\ * Correspondence: mreza.sanaee@gmail.com (M.R.S.); ebertran@ub.edu (E.B.); \\ Tel.: +98-917-300-7254 (M.R.S.); +34-934-021135 (E.B.)
}

Academic Editor: Jordi Puiggalí

Received: 15 November 2016; Accepted: 20 December 2016; Published: 26 December 2016

\begin{abstract}
Size control of core@shell nanostructures is still a challenge. Carbon encapsulated iron nanoparticles (CEINPs) were synthesized by arc discharge plasma method in this study. CEINPs size can be controlled by varying gas composition, due to change in plasma properties. The morphology and structural features were investigated using scanning electron microscopy, transmission electron microscopy (TEM) and high-resolution TEM. Magnetic properties were studied to confirm the changes in CEINPs size by using superconducting quantum interference device. In order to evaluate the carbon shell protection and ensure the absence of iron oxide, selected area electron diffraction technique, energy-dispersive $x$-ray spectroscopy and electron energy loss spectroscopy were employed. Moreover, the degree of carbon order-disorder was studied by Raman Spectroscopy. It was concluded that arc discharge method is a suitable technique for precise size control of CEINPs.
\end{abstract}

Keywords: arc discharge; carbon encapsulated iron nanoparticles; superparamagnetic nanoparticles

\section{Introduction}

Iron nanoparticles (INPs) comprise an important class of nanobiomaterials and have many potential applications in resonance imaging contrast enhancement, tissue repair, drug delivery, cell separation, hyperthermia [1], polymerase chain reaction [2], tissue engineering [3] and deoxyribonucleic acid detection [4]. Moreover, INPs exhibit great potential for applications including data storage [5], water purification [6] and as catalysis [7]. Stability is one of the main requirements for almost any application of INPs. The instability, large aggregation, and rapid bio-degradation of the pure uncoated (naked) magnetic nanoparticles could be overcome by replacement of the naked magnetic nanoparticles by the encapsulated ones [8]. Coating metallic cores by silica [9], carbon [10,11] and precious-metal [12] are examples of popular inorganic coating approaches. Although, to date, most studies have been focused on the development of polymer or silica protective coatings, recently carbon-coated magnetic nanoparticles are receiving more attention, because carbon-based materials have many advantages over polymer or silica, such as high chemical and thermal stability, better conductivity, as well as biocompatibility of carbon-based materials [13]. It is noteworthy that in many cases the protecting shells not only stabilize the nanoparticles, but can also be used for further functionalization, for instance with other nanoparticles or various ligands, depending on the desired 
application [14]. Consequently, synthesis of carbon encapsulated iron nanoparticles are of interest due to their unique properties and potential applications.

Thus far, several smart strategies have been employed to prepare carbon encapsulated iron, nickel, cobalt and magnetic oxides nanoparticles including combustion method [15], hydrothermal reaction [16], detonation synthesis [17], chemical vapor condensation [18], electron beam irradiation [19], laser ablation [20] arc discharge [21], co-carbonization [22] and thermal decomposition [23]. In contrast, these synthesis methods have particular advantages according to their applications. The two important carbon based materials, fullerenes and nanotubes were obtained by arc discharge method [24,25]. CEINPs (carbon encapsulated iron nanoparticles) can be produced in a single step by arc discharge method. This means that the iron particles can be covered by carbon during the growth process. Because no lengthy chemical process is involved in the arc discharge method, it can be easily implemented and adapted for mass production [26]. The advantage of the arc-plasma over other common techniques for the production of CEINPs, such as chemical vapor deposition or laser ablation, is the feasibility to produce high-pure nanoparticles with good surface activity, showing very small iron sizes due to the short residence times of the nuclei in the plasma and the high temperatures used as well as the high cooling rates. In previous work, Aguiló-Aguayo and coworkers showed the difference in morphology/composition of CEINPs obtained with arc-plasma and chemical vapor deposition. As a consequence of that difference, a distinct thermal-induced behavior of the CEINPs was observed [27].

Combustion synthesis is autothermal process and has inherent advantages, including the use of low cost materials and the simplicity of the production protocol [28]. However, this method seems not suitable for encapsulating metallic nanoparticles by carbon. Borysiuk et al. produced and compared CEINPs by using both arc plasma and combustion synthesis methods [29]. According to their experiments, the combustion sample has much higher content of carbon, indicating that the Fe particles were not covered by graphite layer totally, and were dissolved in the etching process. In contrast the Fe-containing particles are much more abundant in arc-plasma samples and they are covered by thicker graphite layers, effectively protecting them during etching process [29]. A hydrothermal process has been reported by Wang et al., they found that only Fe nanoparticles did not favor the formation of CEINPs due to the oxidation of Fe nanoparticle by $\mathrm{H}_{2} \mathrm{O}$ during the reaction [30]. However, maintaining and or increasing quality in parallel with quantity of nanoparticles needs to be improved by arc discharge method. Moreover, plasma instability, high temperature and chemical pollution during the process are drawbacks examples of arc discharge technique. Common objectives of current synthesis methods are improving uniformity, enhancing coating protection and controlling particles composition, shape and size. In addition, due to the lack of comprehensive understanding of the optimal parameters and formation mechanism most of the current fabrication processes are empirical, which means a large number of experimental trials are required to optimize any given process.

In the present study, a modified arc-discharge reactor (patented) has been employed [31]. CEINPs were obtained using different gas compositions to form plasma. All the other parameters were kept at optimum values based on previous experiments [32-34]. Accordingly, the objective is to study influences of argon/helium arc plasma on CEINPs morphology and magnetic properties. Controlling CEINPs size is an important issue for a vast range of applications from nano-scale electronic devices [35] to biomedical applications such as drug delivery, hyperthermia and MRI imaging [36,37]. The control over the iron size distribution and composition is especially important for biomedical applications where superparamagnetic nanoparticles are required. High-pure alpha-iron cores are required to exhibit high saturation magnetization in comparison with iron oxides or iron carbides. In addition, iron particles smaller than $10 \mathrm{~nm}$ are needed to exhibit superparamagnetism at room temperature.

CEINPs are attractive due to their stable characteristics under harsh conditions (e.g., under acidic or basic conditions, reactive atmospheres or high temperatures) [38]. Moreover, the carbon shell of CEINPs can be functionalized, for instance with surface acidic groups in order to investigate the 
treatment of melanoma cancer [39]. It is worth mentioning that nanoparticles can control the basic functions of cells, and potentially kill cancer cells, by virtue of their size alone without the need for drugs [40] because nanoparticles of different sizes are taken up by cancer cells with different efficiency. Potentially, the morphology and magnetic properties of CEINPs shown in this research work are suitable for biomedical applications.

\section{Materials and Methods}

\subsection{Synthesis of CEINPs}

CEINPs were synthetized by a modified arc discharge apparatus [31]. Arc discharge schematic diagram is shown in Figure 1 [34]. The two existing 99.99\% pure graphite rods serve as anode and cathode inside the glass chamber. Cathode graphite rod was sharpening at an angle near to $20^{\circ}$ and placed almost perpendicular to the anode electrode as shown in the inset image of Figure 1. Each graphite rod was fixed to one stainless steel rod. The stainless steel rods are rotatable in vertical axis from outside of the glass chamber. The synthesis and collection process was taken place inside a spherical glass chamber. In order to ensure the absence of oxygen, a rotary vane pump was used for making vacuum inside the glass chamber down to a pressure of $1 \mathrm{~Pa}$. Afterward, Ar and/or $\mathrm{He}$ gas mixtures ([Ar]/[Ar + He]) as the ratios of 1, 0.75, 0.50, 0.25 and 0 were introduced at set flow rate. Based on the utilized gas percentage, the CEINPs samples are named as presented in Table 1. The pressure was always kept at near atmospheric pressure condition by a micro valve connected to a pump. An arc was generated between anode and cathode by a programmable constant power supply which was set at $40 \mathrm{~A}$ for each production run of $5 \mathrm{~min}$ (the voltage was varied about $18 \mathrm{~V}$ ). Precursor consists of ferrocene $(0.5 \mathrm{wt} \%)$ dissolved in isooctane. Ferrocene is a solid organometallic compound which should be dissolved to use in a liquid state. A syringe pump along with an injector regulates the droplets flow of precursor to the plasma zone (droplet flow rate $1 \mathrm{~mL} / \mathrm{min}$ ). Finally, obtained particles were separated through a magnetic filtration.

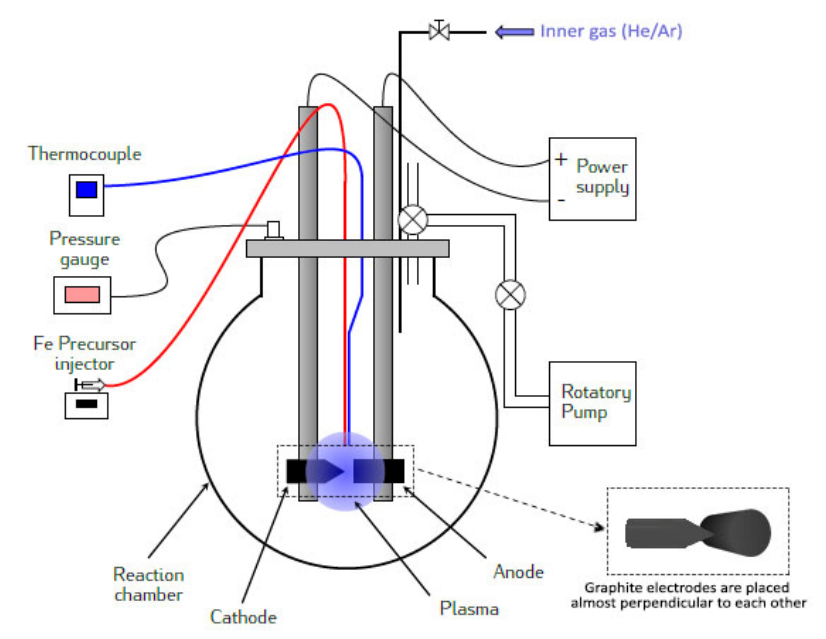

Figure 1. Schematic diagram of arc discharge setup.

Table 1. Samples names and corresponding gas condition.

\begin{tabular}{cccccc}
\hline Sample Name & Ar100 & Ar75 & Ar50 & Ar25 & Ar0 \\
\hline Gas $\%$ & $100 \% \mathrm{Ar}$ & $75 \% \mathrm{Ar}+25 \% \mathrm{He}$ & $50 \% \mathrm{Ar}+50 \% \mathrm{He}$ & $25 \% \mathrm{Ar}+75 \% \mathrm{He}$ & $0 \% \mathrm{Ar}+100 \% \mathrm{He}$ \\
\hline
\end{tabular}

\subsection{Characterization}

CEINPs morphology have been characterized by Jeol JSM 7100F , Jeol JEM 2100, Jeol JEM 2010F (equipped with GIF spectrometer) (JEOL Ltd., Akishima, Japan) and Hitachi MT800 (equipped with 
Bruker XFlash detector 4010 (Hitachi High-Tech Manufacturing \& Service Corp., Ibaraki, Japan) for scanning electron microscopy (SEM), high resolution transmission electron microscopy (HRTEM), and transmission electron microscopy (TEM) images. TEM was equipped with electron energy-loss spectroscopy (EELS) and energy dispersive X-ray (EDX). The magnetic properties of the CEINPs have been investigated by a superconducting quantum interference device (SQUID). The SQUID model was MPMS XL-5 (Quantum Design International, San Diego, CA, USA). The degree of carbon shell order-disorder was studied by Horiba RAMAN spectroscopy equipped with laser detector synapse CCD (HORIBA Scientific, Edison, NJ, USA).

\section{Results and Discussion}

CEINPs have been synthesized using different gas composition by arc discharge method. The samples name and gas composition condition are presented in Table 1. The morphology of CEINPs was studied by SEM, TEM and HRTEM. A representative SEM and TEM images in Figure 2a,b show CEINPs in agglomeration form. HRTEM image in Figure 2c clarifies a clear core@shell structure and sharp interface between them and carbon shell hermetically closed the iron core. It was observed that the structures of CEINPs are almost spherical. TEM and HRTEM images are in agreement with reported images of CEINPs core/shell nanostructure in the literature [41-43]. The CEINPs sizes were determined from the data obtained by SEM, TEM and HRTEM micrographs using ImageJ (1.50i, National Institute of Health, Bethesda, MD, USA). Moreover, iron nanoparticles of CEINPs were identified from HRTEM images and coefficient of variation were calculated using data analysis and Graphing Software-OriginLab (b9.3.226, OriginLab Corporation, Northampton, MA, USA). Coefficients of variation for iron nanoparticles are shown in Table 2. Ar100 sample shows more monodispersion size distribution comparing to other samples (the mean value of the Fe core size is $3.4 \mathrm{~nm}$ ). The Ar100 size distribution histogram is presented in Figure 3. This result suggests the use of argon over helium in large scale production not only due to the monodispersion of CEINPs but also because of availability and cost reason.

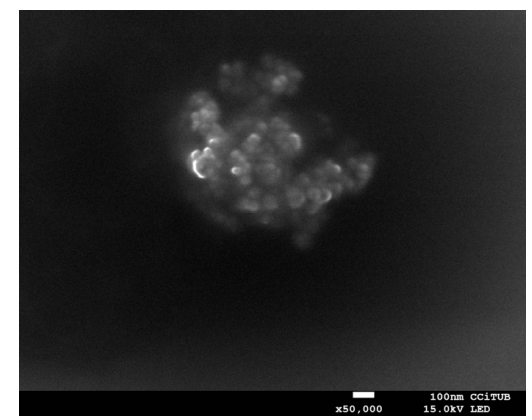

(a)

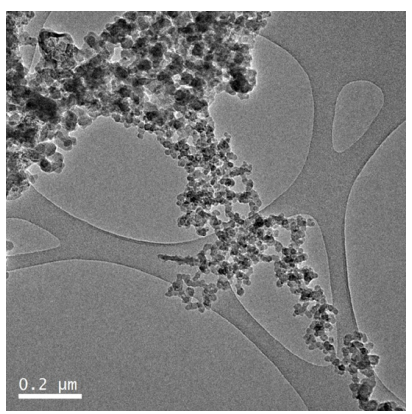

(b)

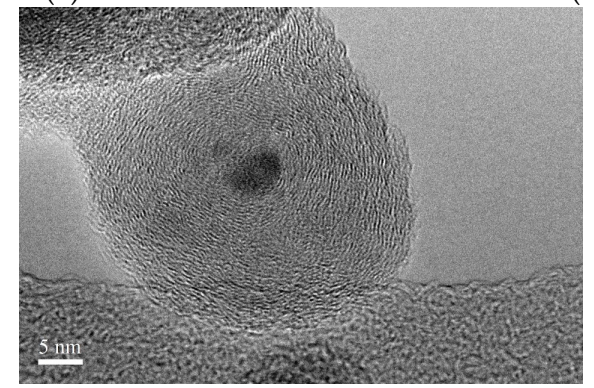

(c)

Figure 2. (a,b) SEM (scanning electron microscopy) and TEM (transmission electron microscopy) images of CEINPs (carbon encapsulated iron nanoparticles), respectively; and (c) HRTEM (high resolution transmission electron microscopy) image from a single iron core at carbon shell nanostructure, the iron core is visible in black spot. 


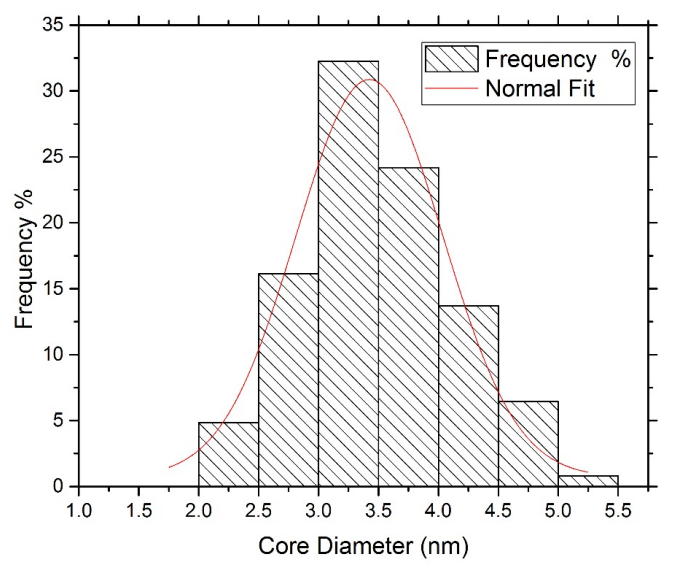

Figure 3. Size distribution histogram of iron core when pure argon is used.

Table 2. This table contains the iron core coefficient of variation (CV) for each simple.

\begin{tabular}{cccccc}
\hline CEINPs ${ }^{1}$ Sample & Ar100 (Pure Argon) & Ar75 & Ar50 & Ar25 & Ar0 (Pure Helium) \\
\hline $\mathrm{CV}$ & 0.17 & 0.21 & 0.30 & 0.22 & 0.21 \\
\hline \multicolumn{6}{c}{ CEINPs: carbon encapsulated iron nanoparticles. }
\end{tabular}

CEINPs HRTEM images are shown in Figure 4. HRTEM images reveal influences of argon/helium plasma on CEINPs morphology. It is evident that the diameters of CEINPs increases with increasing argon gas concentration and diameters of iron nanoparticles (core) decreases with increasing argon gas concentration. Size variation of CEINPs shows, if we consider equal volume of each sample the iron concentration decrease from Ar0 to Ar100.

In addition to point to point observations by TEM and HRTEM, magnetic properties of CEINPs were considered in order to confirm morphology changes. SQUID device were utilized to characterize CEINPs magnetic properties. In principle, magnetic nanoparticles have attracted attention because of the possibility of manipulating them by an external magnetic force. Superparamagnetic behavior provides multifunctional effects such as controlled heating capability under an alternating magnetic field, which is suitable for hyperthermic treatment of cancer [44]. The superparamagnetic state is defined for a system of no interacting single domain nanoparticles, whose susceptibility follows the Curie law, and its magnetization curve can be described by the Langevin function $[45,46]$. Due to the unique core@shell structure of CEINPs, the carbon shell avoid interaction between iron nanoparticles. The normalized magnetizations $M / M_{S}$ as a function of $H / T$ ratio together with the Langevin fitting of the data for all samples at room temperature $(300 \mathrm{~K})$ have been analyzed. Accordingly, effective moment has been calculated. Figure 5 is an example graph showing that these particles have a coercivity close to zero and have no hysteresis. The ratio of remnant to saturation magnetization at room temperature ranges from $M_{r} / M_{s} \sim 0.020$ to 0.05 which clearly indicates that all the samples are in a superparamagnetic state. Figure 6 summarizes the dependence of normalized moment $(\mu)$ and saturation magnetization $(\mathrm{emu} / \mathrm{g})$ on the mean iron core diameter. Saturation magnetization and moment $(\mu)$ is found to increase when the iron nanoparticle size is increasing. These results are in agreement with HRTEM observations. Similar trend and size dependency for iron saturation magnetization have been reported in the literature [47-50]. 


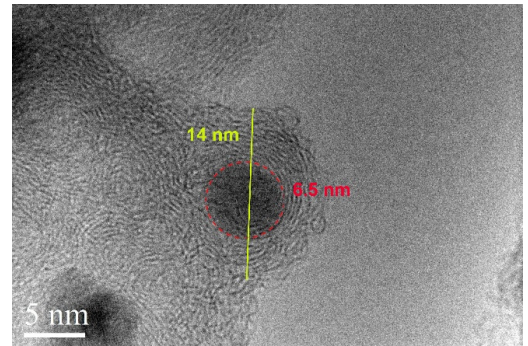

(a) $\mathrm{Ar} 0$

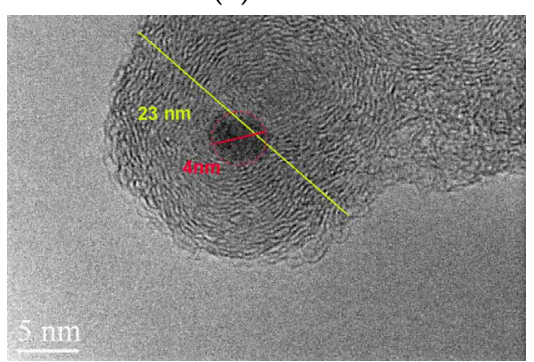

(c) $\operatorname{Ar} 50$

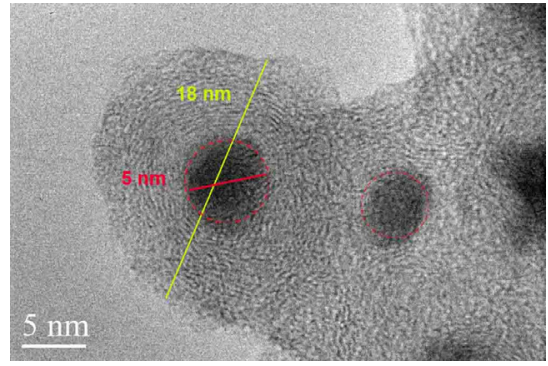

(b) Ar25

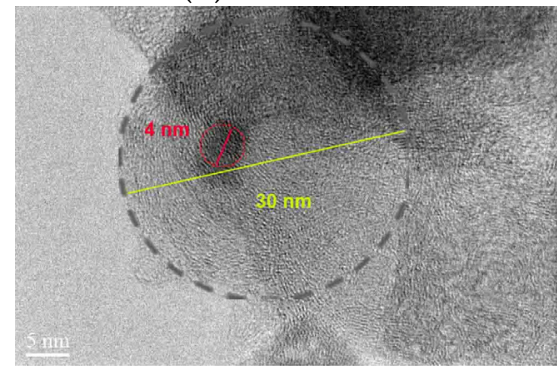

(d) Ar75

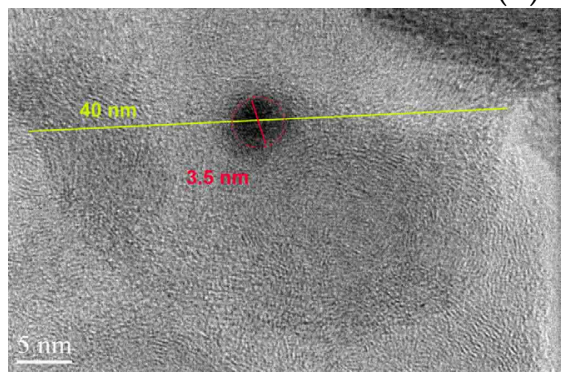

(e) Ar100

Figure 4. The size variations of CEINPs components are evident in HRTEM images. The $100 \%$ helium is used to synthesize Ar0 sample, by adding and increasing $25 \%$ argon, the carbon shell formed larger and iron core formed smaller, as can be observed in Ar25 image, the same trend is shown in Ar50, Ar75, and Ar100 images, respectively. (a) Ar0; (b) Ar25; (c) Ar50; (d) Ar75; (e) Ar100.

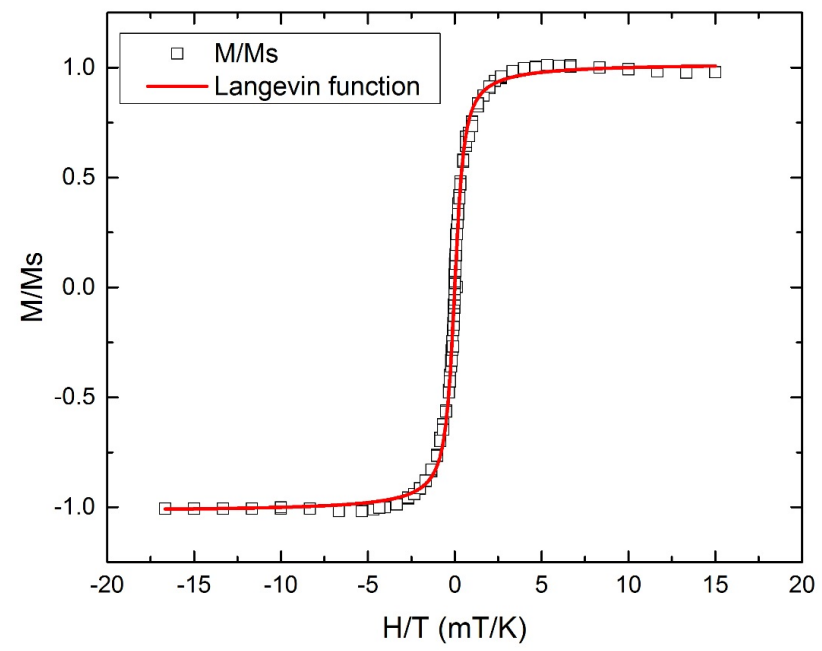

Figure 5. Temperature magnetization data to saturation magnetization $M_{S}$, are reported as a function of the $H / T$ ratio together with the Langevin fit of the data. 


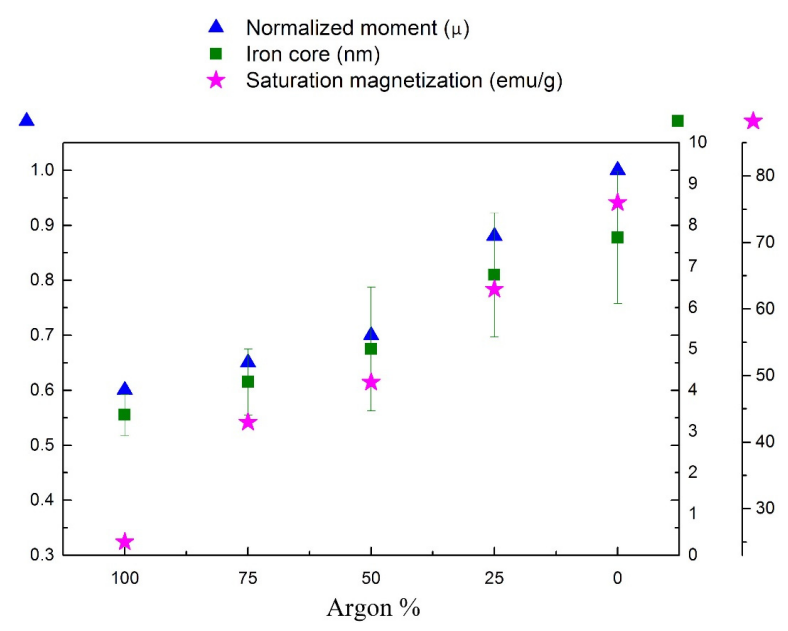

Figure 6. Effects of iron core sizes on normalized moment $(\mu)$ and saturation magnetization (emu/g) versus argon gas percentage. The error bars correspond to iron core size dispersion.

Zero-field-cooling (ZFC) and field-cooling (FC) magnetization as a function of temperature measured at 100 Oe have been analyzed. ZFC and FC graphs are plotted in Figure 7. The blocking temperature was determined as the peak temperature of the $M(T)$ curve after ZFC [51]. For ZFC curves, as the temperature increases, the magnetization shows an increase because the magnetic moment is thermally activated along the magnetic field direction. For samples Ar100, Ar75, Ar50, Ar25 and Ar0, the transition temperature from ferromagnetic to superparamagnetic state or blocking temperature, $T_{b}$, peaks can be observed in the ZFC curve at about $24 \mathrm{~K}, 26 \mathrm{~K}, 30 \mathrm{~K}, 41 \mathrm{~K}$ and $55 \mathrm{~K}$, respectively. By contrast, the $T_{b}$ value of $\operatorname{Ar} 0$ is almost double of Ar100 $T_{b}$, due to the larger iron particles. This result is in agreement with HRTEM observations of iron core diameter. The finding that $T_{b}$ increases as the size of the nanoparticles increases is consistent with conventional Stoner-Wohlfarth theory: the energy barrier, over which the magnetization of the nanoparticles should be thermally activated, increases as the size of the nanoparticles increases [52]. The sizes of iron core nanoparticles were estimated from HRTEM images. Dependency between $\mathrm{Tb}$ and iron core size is plotted in Figure 8 . From the results obtained, it is concluded that the gas composition used in the arc-plasma can have an influence on the Fe core size distribution as well as total size of the CEINPs. Above blocking temperature, the particles are free to align with the field during the measuring time and ZFC curve superimposes with FC curve, this state is called superparamagnetic, because the particle behaves similarly to paramagnetic spin but with a much larger moment [53]. ZFC and FC curves demonstrate that all samples are in superparamagnetic state above $260 \mathrm{~K}$. Ar100 sample is superparamagnetic at low temperature (above $220 \mathrm{~K}$ ), therefore it is a suitable CEINPs for specific applications that operate at extremely low temperature such as applications in aerospace industry.

The inert gas to generate plasma appears to be a sensitive parameter to control CEINPs size. The synthesis of nanoparticles by thermal plasmas is an intricate heat and mass transfer process that involves a phase conversion in a few tens of milliseconds, as well as interactions between the thermofluid field, the induced electromagnetic field and the particle concentration field, all of which are described by numerous variables [54]. Despite the strong industrial and scientific interest in the topic, the growth mechanism of the nanoparticles remains poorly understood [54].

The mechanisms for the formation of CEINPs depend on the fabrication technique. Concerning the arc-discharge plasma methods, the detailed pathways of the mechanisms remain unclear. Saito et al. observed a correlation between the vapor pressure of the metal and the graphite encapsulation [55]. Majetich et al. suggested that for the formation of CEINPs, the atomization of the core materials and carbon occurred firstly in the plasma [56,57]. Seraphin et al. observed experimentally that the enthalpy of the carbide formation was decisive for the carbon encapsulation [58]. However, they also observed 
the exception for some core elements. Bystrzejewski et al. proposed another mechanism where the formation of $\alpha$-Fe from the vapor phase under a dominating presence of carbon was not possible [59]. They suggested that CEINPs emerged from liquid droplets and then solidified in the cold zone. A more systematic research is required for a clear understanding of the mechanisms of the CEINPs formation.
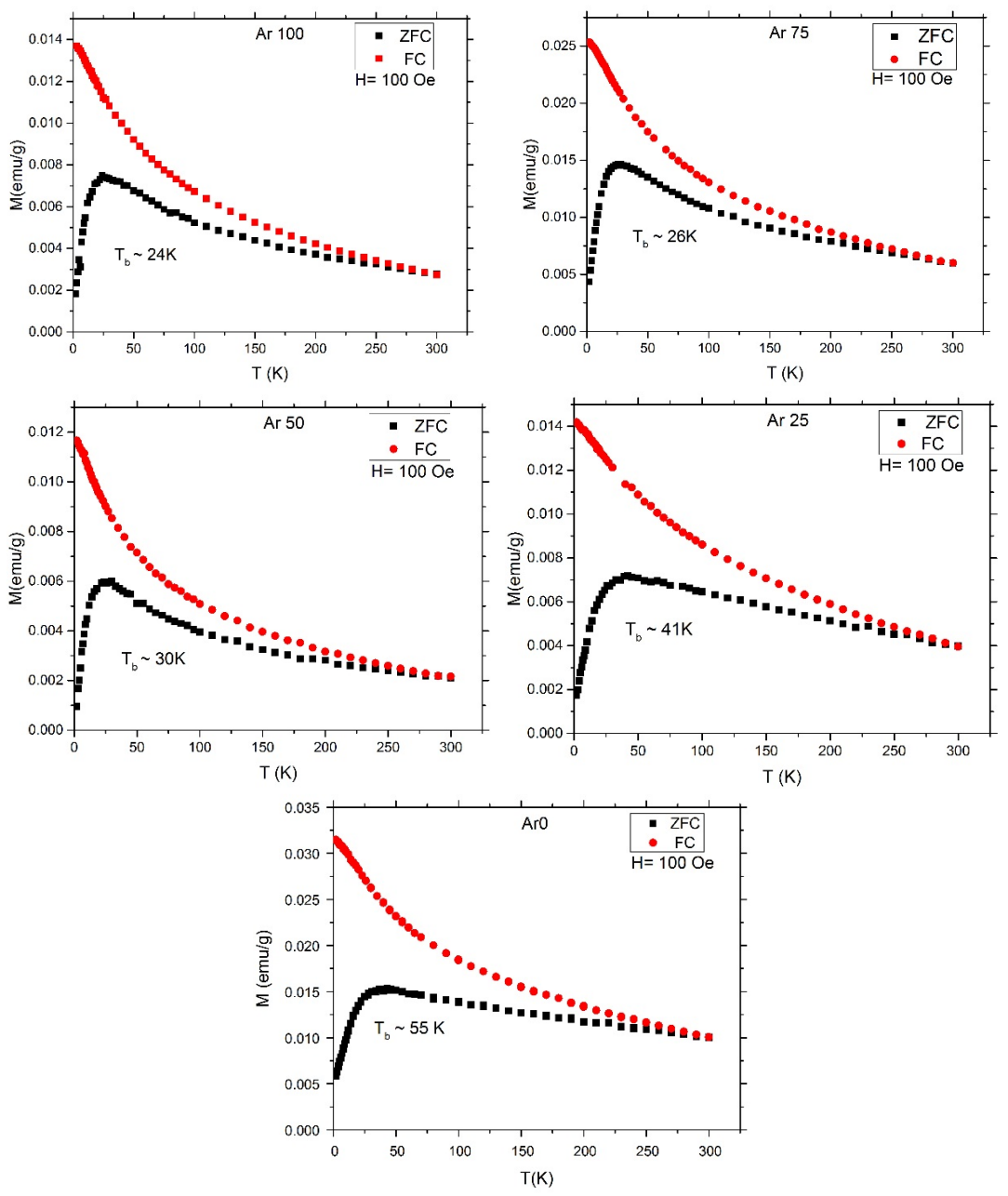

Figure 7. Zero-field-cooled and $(H=100$ Oe) field-cooled magnetization curves of samples: Ar100, Ar75, Ar50, Ar25 and Ar0.

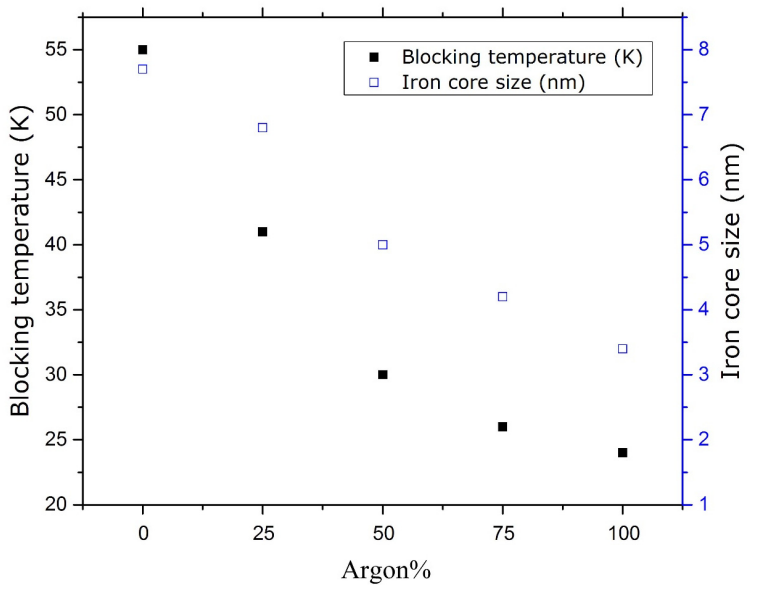

Figure 8. Dependency of iron core size and blocking temperature versus argon percentage. 
According to previous studies [32-34], optimum parameters are kept constant and only influences of the Argon/Helium and their mixtures are studied to explain growth mechanism from this perspective. Four major phases are considered to describe the formation of CEINPs: evaporation, expansion, growth of iron core and carbon shell. The processes start with vaporization of precursor. The precursor is in the form of a droplet pass through plasma spot between carbon rods of cathode and anode. Cracking of molecules occurred in expansion phase and carbon, iron and hydrogen are available. Haines and co-workers concluded that the sublimation rate of graphite rods under static argon is about two times higher than static helium $[60,61]$. Due to the higher temperature of argon plasma comparing to helium plasma, more carbon species are available in expansion phase for carbon particles formation.

Nanoparticles rapid production takes place in supersaturated vapor condition. Although inert gases allow any required chemical reactions to occur, they affect the plasma formation and consequently change temperature gradient of plasma. It should also be considered that thermal conductivity of argon is about eight times smaller than helium [62]. Varying the argon-helium gas ratio alters the heat transfer from central of the plasma to outside, when argon concentration increases then temperature gradient increases or in other words cooling rate decreases, as the result it changes the critical radius of carbon shell and iron core particles.

CEINPs are formed through heterogeneous nucleation, which involves formation of a carbon phase (shell) on the surface of iron nanoparticles (core). Heterogeneous nucleation involves decrease in the Gibbs free energy and an increase in surface of interface energy [63]. The nucleus is stable only when its size is larger than a critical diameter. The critical diameter for the formation of a stable iron nucleus depends on the temperature inside the plasma. Higher temperatures allow the formation of smaller iron cores. The use of argon increases the temperature inside the plasma. This could be the mechanism to obtain larger total sizes of the nanoparticles (thicker carbon thicknesses), since higher carbon amounts are present inside the plasma. In addition, the presence of high amounts of carbon might reduce the interaction between the iron cores and limit coalescence among them, promoting smaller iron core sizes. Nanoparticle growth occurs in gas phase [56] and it seems that lower thermal conductivity of argon prolonged the formation time. Accordingly, the CEINPs sizes were changed in this experimental study.

Naked metallic nanoparticles are highly chemical active and easy to be oxidized in air, which results in poor magnetism and dispersibility [64]. One of the advantages of arc discharge technique is possibility of protecting iron nanoparticles by carbon shell in one step. Due to the incomplete carbon shell, the iron cores will oxidize rapidly in contact with air. In this case the carbon shell surface cannot be functionalized properly and their efficiency will be low. For this reason, three methods, selected area electron diffraction (SAED), EELS and EDX, were used to evaluate the carbon shell protection against iron core oxidation. Primarily SAED technique was used to verify iron oxidation issue. The SAED pattern in Figure 9 for CEINPs were successfully indexed to iron $(\alpha$-Fe) crystal structure as identified by diffraction rings corresponding to the (110) and (112) hkl planes. Electron diffraction from iron oxides was not detected in any of our samples. In general, it should be noted that SAED images of CEINPs reveal diffuse rings that are due to the presence of both nanocrystalline and amorphous phases of carbon.

In addition, several points of CEINPs have been characterized by EDX analysis at micro scale and EELS analysis at nanoscale. An example of elemental and composition mapping along with corresponding sample image are presented in Figure 10. As it is shown in Figure 11, detailed results of EELS are in agreement with EDX analysis results. Consequently, only iron peak has been observed from both EELS and EDX analysis and no trace of oxygen or other impurities have been found which shows that all iron particles were well protected by carbon shells.

More detailed information of carbon shell characteristics can be achieved by Raman spectroscopy; thereby the degree of carbon order-disorder is studied. The Raman spectra are fitted with Lorentzian function for further analysis. Example spectrum is presented in Figure 12. The G-line and D-line Raman spectra peaks are observed around $1600 \mathrm{~cm}^{-1}$ and $1350 \mathrm{~cm}^{-1}$ respectively. The peak intensity ratio 
$I_{D} / I_{G}$ is often used to determine the extent of structural disorder (e.g., $I_{D} / I_{G}=0$ for a perfect, infinite graphene layer) in graphite and/or the size of the graphitic domains [65]. As shown in Figure 13, the ratio $I_{D} / I_{G}$ is decreasing as the carbon thickness of iron core is getting larger and exhibit higher degree of graphitization. This is probably due to the lower cooling rate of vapor when plasma is generated by argon in comparison to helium plasma. Laplaze et al. pointed out the great influence of the cooling rate of vapors on the structure and yield of nanostructured carbon material; they observed a fast cooling rate when plasma is generated by helium and lower with argon at close flow rate [66]. Raman spectroscopy results indicate that the crystallinity of the carbon shell increases when pure argon is used.

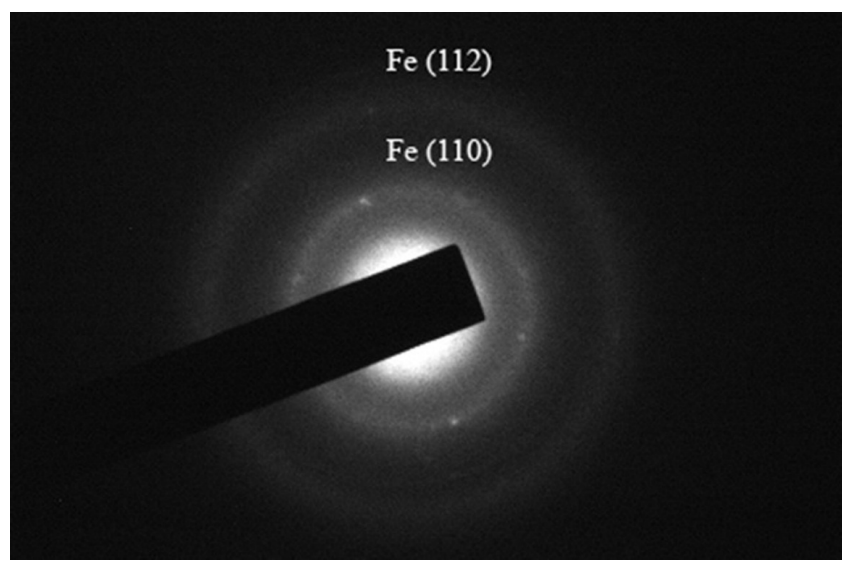

Figure 9. SAED (selected area electron diffraction) pattern showed pure iron phases, no diffraction point of iron oxides was detected.

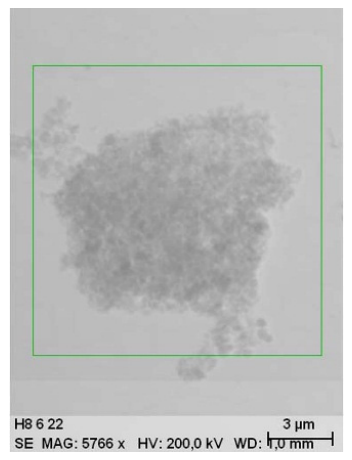

(a)

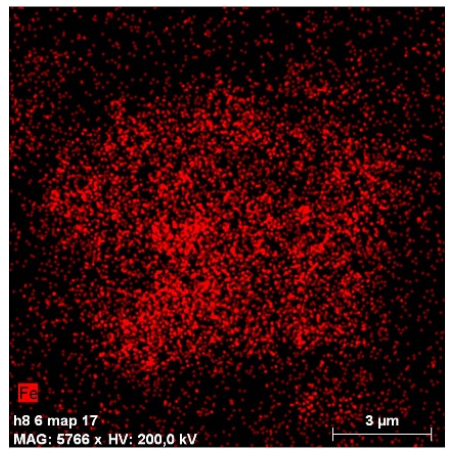

(b)

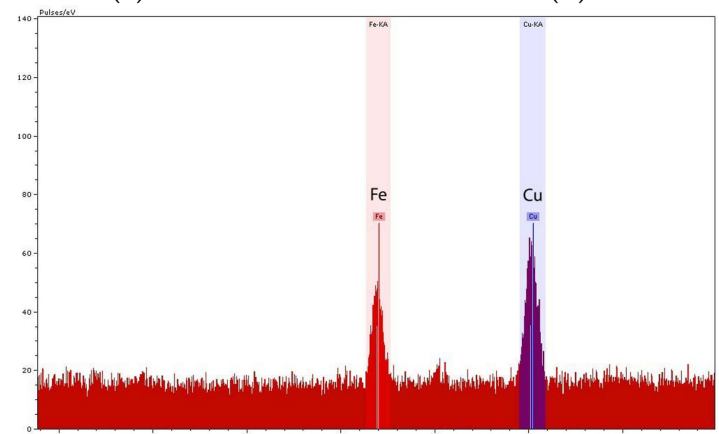

(c)

Figure 10. (a) TEM image at micro scale; (b) chemical mapping in conjunction with TEM image (a), red points accumulation represent appearance of iron in particles; (c) EDX (energy dispersive X-ray) spectrum, red peak corresponds to $\mathrm{Fe}$ and blue peak is $\mathrm{Cu}$ from grid of the sample. 


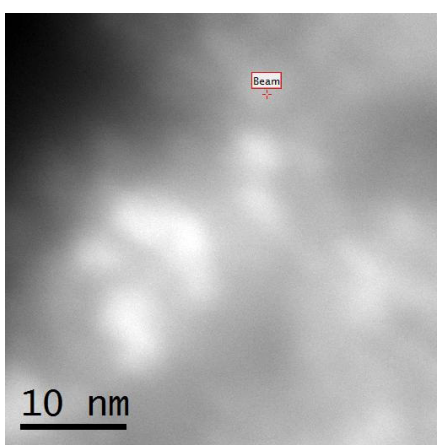

(a)

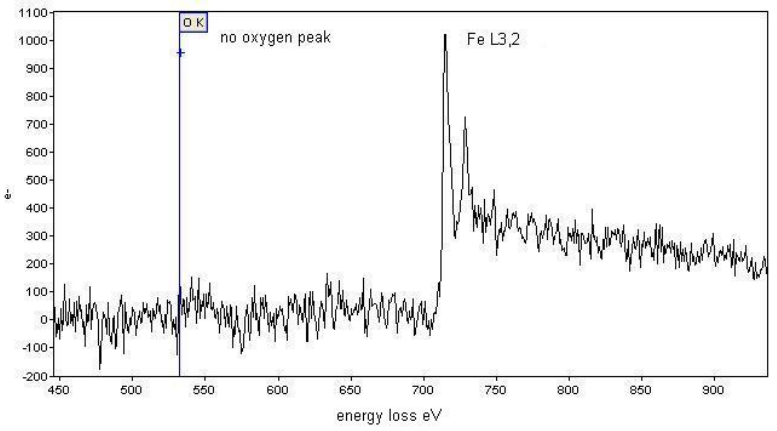

(b)

Figure 11. (a) An example of TEM image from EELS (electron energy-loss spectroscopy) analysis; (b) EELS spectrum from image (a) (only iron peaks were detected).

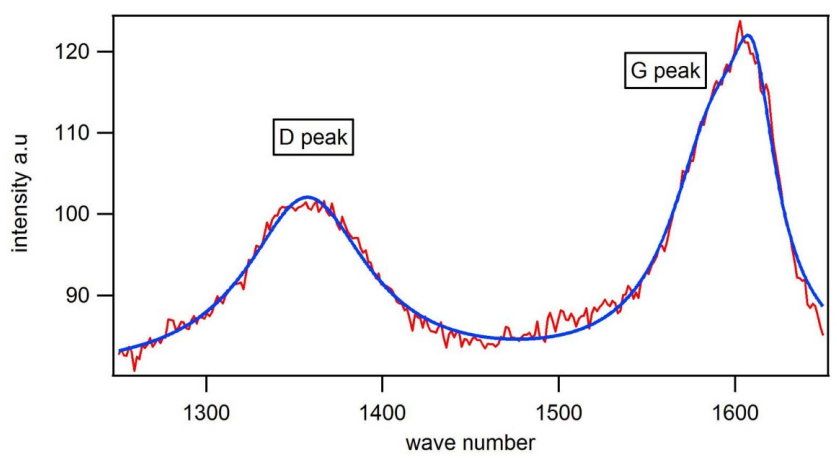

Figure 12. Raman spectrum fitted to Lorentzian function, $I_{D} / I_{G}<1$.

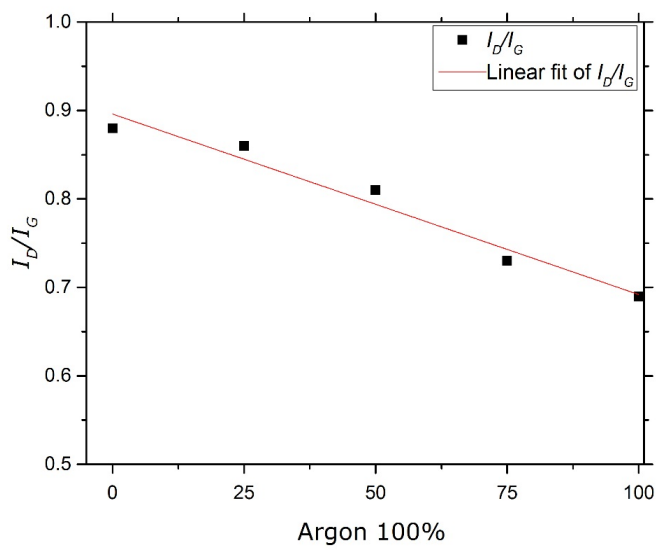

Figure 13. $I_{D} / I_{G}$ packs vs. argon percentage.

\section{Conclusions}

Carbon encapsulated iron nanoparticles were synthesized successfully in a controlled manner. CEINPs characterization demonstrated that by changing gas composition of the arc discharge reactor used in the research project, morphological properties of carbon encapsulated iron nanoparticles can be controlled. In addition to SEM, TEM and HRTEM observations, the analysis of effective moment, saturation magnetization and blocking temperature reveals the size changes of CEINPs. It is concluded that by increasing ratios of argon to helium, carbon shell thickness increases and iron core decreases. Diffraction points of iron oxide were not detected from SAED analysis. Moreover, both EELS analysis at nanoscale and EDX analysis at micro scale showed no trace of oxygen in iron cores. 
These results point to a high efficiency of carbon shell to isolate iron core from a possible external oxidation. According to Raman spectroscopy results crystallinity of the carbon shell increases when pure argon is used. All studied CEINPs exhibit superparamagnetic behavior above $260 \mathrm{~K}$. The results of this study could be helpful in interpreting growth mechanism and controlling carbon and iron core/shell nanostructure sizes using arc discharge method.

Acknowledgments: We acknowledge Sonia Estrade, Lluís Yedra, Joan Mendoza and Núria Clos of Centres Científics i Tecnològics of the Universitat de Barcelona (CCIT-UB) for electronic microscopy facilities and SQUID measurements. This work was partially financed by the Ministerio de Ciencia e Innovación of Spanish Government, under contract MAT2010 20468, and by Agència de Gestió d'Ajuts Universitaris i de Recerca of Generalitat de Catalunya, under contract 2009SGR00185. The first author also acknowledges the Iranian National Elites Foundation for their support.

Author Contributions: Mohammad Reza Sanaee conceived, performed the experiments and wrote the manuscript. Stefanos Chaitoglou contributed to the performing the experiments. Noemi Aguiló-Aguayo contributed to the study design and Enric Bertran supervised the study. All authors have reviewed and approved the final manuscript.

Conflicts of Interest: The authors declare no conflict of interest.

\section{References}

1. Iacovita, C.; Florea, A.; Dudric, R.; Pall, E.; Moldovan, A.I.; Tetean, R.; Stiufiuc, R.; Lucaciu, C.M. Small versus large iron oxide magnetic nanoparticles: Hyperthermia and cell uptake properties. Molecules 2016, 21. [CrossRef] [PubMed]

2. Higashi, T.; Minegishi, H.; Nagaoka, Y.; Fukuda, T.; Echigo, A.; Usami, R.; Maekawa, T.; Hanajiri, T. Effects of superparamagnetic nanoparticle clusters on the polymerase chain reaction. Appl. Sci. 2012, 2, 303-314. [CrossRef]

3. Popa, E.G.; Santo, V.E.; Rodrigues, M.T.; Gomes, M.E. Magnetically-responsive hydrogels for modulation of chondrogenic commitment of human adipose-derived stem cells. Polymers 2016, 8. [CrossRef]

4. Li, Y.; Burke, D.T.; Kopelman, R.; Burns, M.A. Asynchronous magnetic bead rotation (AMBR) microviscometer for label-free DNA analysis. Biosensors 2014, 4, 76-89. [CrossRef] [PubMed]

5. Singamaneni, S.; Bliznyuk, V.N.; Binek, C.; Tsymbal, E.Y. Magnetic nanoparticles: Recent advances in synthesis, self-assembly and applications. J. Mater. Chem. 2011, 21, 16819-16845. [CrossRef]

6. Lemos, B.R.; Teixeira, A.P.C.; Ardisson, J.D.; Macedo, W.A.; Fernandez-Outon, L.E.; Amorim, C.C.; Moura, F.C.; Lago, R.M. Magnetic amphiphilic composites applied for the treatment of biodiesel wastewaters. Appl. Sci. 2012, 2, 513-524. [CrossRef]

7. Yepez, A.; De, S.; Climent, M.S.; Romero, A.A.; Luque, R. Microwave-assisted conversion of levulinic acid to $\gamma$-valerolactone using low-loaded supported iron oxide nanoparticles on porous silicates. Appl. Sci. 2015, 5, 532-543. [CrossRef]

8. Galakhov, V.; Shkvarin, A.; Semenova, A.; Uimin, M.; Mysik, A.; Shchegoleva, N.; Yermakov, A.Y.; Kurmaev, E. Characterization of carbon-encapsulated nickel and iron nanoparticles by means of $\mathrm{x}$-ray absorption and photoelectron spectroscopy. J. Phys. Chem. C 2010, 114, 22413-22416. [CrossRef]

9. De Matteis, L.; Fernandez-Pacheco, R.; Custardoy, L.; García-Martín, M.L.; de la Fuente, J.S.M.; Marquina, C.; Ibarra, M.R. Influence of a silica interlayer on the structural and magnetic properties of sol-gel $\mathrm{TiO}_{2}$-coated magnetic nanoparticles. Langmuir 2014, 30, 5238-5247. [CrossRef] [PubMed]

10. Li, S.-S.; Chiu, C.-C.; Chang, R.-W.; Liou, Y.-H.; Teng, M.-H. Synthesis and properties of modified graphite encapsulated iron metal nanoparticles. Diam. Relat. Mater. 2016, 63, 153-158. [CrossRef]

11. Sanaee, M.R.; Freire, V.; Aguil, N.; Bertran, E.; Aguiló-Aguayo, N. Design and synthesis of carbon encapsulated iron nanoparticle for drug delivery. In Proceedings of the 10th International Conference on nanoscience and nanotechnology, Bilbao, Spain, 23-26 April 2013; pp. 212-213.

12. Schärtl, W. Current directions in core-shell nanoparticle design. Nanoscale 2010, 2, 829-843. [CrossRef] [PubMed]

13. Lu, A.H.; Salabas, E.E.L.; Schüth, F. Magnetic nanoparticles: Synthesis, protection, functionalization, and application. Angew. Chem. Int. Ed. 2007, 46, 1222-1244. [CrossRef] [PubMed] 
14. Bakthavathsalam, P.; Rajendran, V.K.; Saran, U.; Chatterjee, S.; Ali, B.M.J. Immunomagnetic nanoparticle based quantitative pcr for rapid detection of salmonella. Microchim. Acta 2013, 180, 1241-1248. [CrossRef]

15. Zhao, B.; Yu, X.; Cai, R.; Ran, R.; Wang, H.; Shao, Z. Solution combustion synthesis of high-rate performance carbon-coated lithium iron phosphate from inexpensive iron (III) raw material. J. Mater. Chem. 2012, 22, 2900-2907. [CrossRef]

16. Xuan, S.; Hao, L.; Jiang, W.; Gong, X.; Hu, Y.; Chen, Z. A facile method to fabricate carbon-encapsulated $\mathrm{Fe}_{3} \mathrm{O}_{4}$ core/shell composites. Nanotechnology 2007, 18. [CrossRef] [PubMed]

17. Lu, Y.; Zhu, Z.; Liu, Z. Carbon-encapsulated fe nanoparticles from detonation-induced pyrolysis of ferrocene. Carbon 2005, 43, 369-374. [CrossRef]

18. Wang, Z.; Zhang, Z.; Choi, C.; Kim, B. Structure and magnetic properties of Fe (C) and Co (C) nanocapsules prepared by chemical vapor condensation. J. Alloys Compd. 2003, 361, 289-293. [CrossRef]

19. Nishijo, J.; Okabe, C.; Bushiri, J.; Kosugi, K.; Nishi, N.; Sawa, H. Formation of carbon-encapsulated metallic nano-particles from metal acetylides by electron beam irradiation. Eur. Phys. J. D Atom. Mol. Opt. Plasma Phys. 2005, 34, 219-222. [CrossRef]

20. Park, J.; Jeong, S.; Jeong, M.; Kim, J.; Cho, B. Synthesis of carbon-encapsulated magnetic nanoparticles by pulsed laser irradiation of solution. Carbon 2008, 46, 1369-1377. [CrossRef]

21. Chaitoglou, S.; Sanaee, M.R.; Aguiló-Aguayo, N.; Bertran, E. Arc-discharge synthesis of iron encapsulated in carbon nanoparticles for biomedical applications. J. Nanomater. 2014, 2014. [CrossRef]

22. Song, H.; Chen, X. Large-scale synthesis of carbon-encapsulated iron carbide nanoparticles by co-carbonization of durene with ferrocene. Chem. Phys. Lett. 2003, 374, 400-404. [CrossRef]

23. Chen, C.-J.; Chiang, R.-K.; Wang, J.-S.; Wang, S.-L. Synthesis and magnetic properties of octahedral magnetite nanoparticles in 20-110 nm range. J. Nanopart. Res. 2013, 15. [CrossRef]

24. Ando, Y.; Iijima, S. Preparation of carbon nanotubes by arc-discharge evaporation. Jpn. J. Appl. Phys. Part 2 Lett. 1993, 32. [CrossRef]

25. Saito, Y.; Inagaki, M.; Shinohara, H.; Nagashima, H.; Ohkohchi, M.; Ando, Y. Yield of fullerenes generated by contact arc method under He and Ar: Dependence on gas pressure. Chem. Phys. Lett. 1992, 200, 643-648. [CrossRef]

26. Fang, F.; Kennedy, J.; Futter, J.; Markwitz, A.; Manikandan, E. Transition metal doped metal oxide nanostructures synthesized by arc discharge method. In Proceedings of the 2013 International Conference on Manipulation, Manufacturing and Measurement on the Nanoscale (3M-NANO), Suzhou, China, 26-30 August 2013; pp. 220-223.

27. Aguiló-Aguayo, N.; Liu, Z.; Bertran, E.; Yang, J. Thermal-induced structural evolution of carbon-encapsulated iron nanoparticles generated by two different methods. J. Phys. Chem. C 2013, 117, 19167-19174. [CrossRef]

28. Bystrzejewski, M.; Huczko, A.; Lange, H.; Cudziło, S.; Kiciński, W. Combustion synthesis route to carbon-encapsulated iron nanoparticles. Diam. Relat. Mater. 2007, 16, 225-228. [CrossRef]

29. Borysiuk, J.; Grabias, A.; Szczytko, J.; Bystrzejewski, M.; Twardowski, A.; Lange, H. Structure and magnetic properties of carbon encapsulated Fe nanoparticles obtained by arc plasma and combustion synthesis. Carbon 2008, 46, 1693-1701. [CrossRef]

30. Wang, Z.; Xiao, P.; He, N. Synthesis and characteristics of carbon encapsulated magnetic nanoparticles produced by a hydrothermal reaction. Carbon 2006, 44, 3277-3284. [CrossRef]

31. Bertran-Serra, E.; Inestrosa-Izurieta, M. Method and Reactor for the Production of Carbon-Coated Nanoparticles. Patent 2,012,025,652, 1 March 2012.

32. Sanaee, M.R. Synthesis and Characterization of Engineered Carbon-Based Nanoparticles by arc-Discharge Plasma; Universitat de Barcelona: Barcelona, Spain, 2015.

33. Sanaee, M.R.; Bertran, E. Synthesis of carbon encapsulated mono- and multi-iron nanoparticles. J. Nanomater. 2015, 2015. [CrossRef]

34. Aguayo, N.A. Production and Characterisation of Carbon-Encapsulated Iron Nanoparticles by arc-Discharge Plasma. Ph.D. Thesis, Universitat de Barcelona, Barcelona, Spain, 2012.

35. Thomas, R.; Swathi, R. Organization of metal nanoparticles for surface-enhanced spectroscopy: A difference in size matters. J. Phys. Chem. C 2012, 116, 21982-21991. [CrossRef]

36. Oh, W.-K.; Yoon, H.; Jang, J. Size control of magnetic carbon nanoparticles for drug delivery. Biomaterials 2010, 31, 1342-1348. [CrossRef] [PubMed] 
37. McDermott, S.; Guimaraes, A.R. Magnetic nanoparticles in the imaging of tumor angiogenesis. Appl. Sci. 2012, 2, 525-534. [CrossRef]

38. Aguiló-Aguayo, N.; Maurizi, L.; Galmarini, S.; Ollivier-Beuzelin, M.G.; Coullerez, G.; Bertran, E.; Hofmann, H. Aqueous stabilisation of carbon-encapsulated superparamagnetic $\alpha$-iron nanoparticles for biomedical applications. Dalton Trans. 2014, 43, 13764-13775. [CrossRef] [PubMed]

39. Grudzinski, I.P.; Bystrzejewski, M.; Cywinska, M.A.; Kosmider, A.; Poplawska, M.; Cieszanowski, A.; Ostrowska, A. Cytotoxicity evaluation of carbon-encapsulated iron nanoparticles in melanoma cells and dermal fibroblasts. J. Nanopart. Res. 2013, 15. [CrossRef] [PubMed]

40. Ferrari, M. Nanogeometry: Beyond drug delivery. Nat. Nanotechnol. 2008, 3, 131-132. [CrossRef] [PubMed]

41. Luo, N.; Li, X.; Wang, X.; Yan, H.; Zhang, C.; Wang, H. Synthesis and characterization of carbon-encapsulated iron/iron carbide nanoparticles by a detonation method. Carbon 2010, 48, 3858-3863. [CrossRef]

42. Huo, J.; Song, H.; Chen, X. Preparation of carbon-encapsulated iron nanoparticles by co-carbonization of aromatic heavy oil and ferrocene. Carbon 2004, 42, 3177-3182. [CrossRef]

43. Zhang, F.; Cui, L.; Lin, K.; Jin, F.-M.; Wang, B.; Shi, S.-X.; Yang, D.-A.; Wang, H.; He, F.; Chen, X.-P. Preparation of carbon-encapsulated iron nanoparticles in high yield by DC arc discharge and their characterization. J. Alloys Compd. 2013, 553, 367-374. [CrossRef]

44. Ruiz-Hernandez, E.; Baeza, A.; Vallet-Regí, M.A. Smart drug delivery through DNA/magnetic nanoparticle gates. ACS Nano 2011, 5, 1259-1266. [CrossRef] [PubMed]

45. Bittova, B.; Vejpravova, J.P.; Kalbac, M.; Burianova, S.; Mantlikova, A.; Danis, S.; Doyle, S. Magnetic properties of iron catalyst particles in HiPco single wall carbon nanotubes. J. Phys. Chem. C 2011, 115, 17303-17309. [CrossRef]

46. Ramírez-Meneses, E.; Betancourt, I.; Morales, F.; Montiel-Palma, V.; Villanueva-Alvarado, C.; Hernández-Rojas, M. Superparamagnetic nickel nanoparticles obtained by an organometallic approach. J. Nanopart. Res. 2011, 13, 365-374. [CrossRef]

47. Seehra, M.; Singh, V.; Dutta, P.; Neeleshwar, S.; Chen, Y.; Chen, C.; Chou, S.; Chen, C.C. Size-dependent magnetic parameters of fcc FePt nanoparticles: Applications to magnetic hyperthermia. J. Phys. D Appl. Phys. 2010, 43. [CrossRef]

48. Dutta, P.; Pal, S.; Seehra, M.; Shah, N.; Huffman, G. Size dependence of magnetic parameters and surface disorder in magnetite nanoparticles. J. Appl. Phys. 2009, 105. [CrossRef]

49. Sanchez, R.; Rivas, J.; Vaqueiro, P.; Lopez-Quintela, M.; Caeiro, D. Particle size effects on magnetic properties of yttrium iron garnets prepared by a sol-gel method. J. Magn. Magn. Mater. 2002, 247, 92-98. [CrossRef]

50. Donegan, K.P.; Godsell, J.F.; Otway, D.J.; Morris, M.A.; Roy, S.; Holmes, J.D. Size-tuneable synthesis of nickel nanoparticles. J. Nanopart. Res. 2012, 14, 670. [CrossRef]

51. Shiratsuchi, Y.; Yamamoto, M.; Endo, Y.; Li, D.; Bader, S. Superparamagnetic behavior of ultrathin fe films grown on $\mathrm{Al}_{2} \mathrm{O}_{3}$ (0001) substrates. J. Appl. Phys. 2003, 94, 7675-7679. [CrossRef]

52. Lee, Y.; Lee, J.; Bae, C.J.; Park, J.G.; Noh, H.J.; Park, J.H.; Hyeon, T. Large-scale synthesis of uniform and crystalline magnetite nanoparticles using reverse micelles as nanoreactors under reflux conditions. Adv. Funct. Mater. 2005, 15, 503-509. [CrossRef]

53. Raj, B. Frontiers in Materials Science; Universities Press: Bangalore, India, 2005.

54. Shigeta, M.; Murphy, A.B. Thermal plasmas for nanofabrication. J. Phys. D Appl. Phys. 2011, 44. [CrossRef]

55. Saito, Y. Nanoparticles and filled nanocapsules. Carbon 1995, 33, 979-988. [CrossRef]

56. Majetich, S.; Scott, J.; Brunsman, E.; McHenry, M. Formation of nanoparticles in a carbon arc. In Proceedings of the MRS Proceedings, San Francisco, CA, USA, 4-8 April 1994; Cambridge University Press: Cambridge, UK; p. 29.

57. Scott, J.H.J.; Majetich, S.A. Morphology, structure, and growth of nanoparticles produced in a carbon arc. Phys. Rev. B 1995, 52. [CrossRef]

58. Seraphin, S.; Zhou, D.; Jiao, J. Filling the carbon nanocages. J. Appl. Phys. 1996, 80, 2097-2104. [CrossRef]

59. Bystrzejewski, M.; Huczko, A.; Lange, H. Arc plasma route to carbon-encapsulated magnetic nanoparticles for biomedical applications. Sens. Actuators B Chem. 2005, 109, 81-85. [CrossRef]

60. Haines, J.; Tsai, C. Graphite Sublimation Tests for the Muon Collider/Neutrino Factory Target Development Program; ORNL/TM-2002/27; Oak Ridge National Laboratory: Oak Ridge, TN, USA, 2002. 
61. Tsai, C.; Gabriel, T.; Haines, J.; Rasmussen, D. Graphite sublimation tests for target development for the muon collider/neutrino factory. In Proceedings of the 21st IEEE/NPS Symposium on Fusion Engineering SOFE 05, Knoxville, TN, USA, 26-29 September 2005; pp. 1-3.

62. Farhat, S.; de La Chapelle, M.L.; Loiseau, A.; Scott, C.D.; Lefrant, S.; Journet, C.; Bernier, P. Diameter control of single-walled carbon nanotubes using argon-helium mixture gases. J. Chem. Phys. 2001, 115, 6752-6759. [CrossRef]

63. Yadav, S.K. Nanoscale Materials in Targeted Drug Delivery, Theragnosis and Tissue Regeneration; Springer: Berlin, Germany, 2016.

64. Peng, R.; Zhang, W.; Ran, Q.; Liang, C.; Jing, L.; Ye, S.; Xian, Y. Magnetically switchable bioelectrocatalytic system based on ferrocene grafted iron oxide nanoparticles. Langmuir 2011, 27, 2910-2916. [CrossRef] [PubMed]

65. Sethuraman, V.A.; Hardwick, L.J.; Srinivasan, V.; Kostecki, R. Surface structural disordering in graphite upon lithium intercalation/deintercalation. J. Power Sources 2010, 195, 3655-3660. [CrossRef]

66. Laplaze, D.; Alvarez, L.; Guillard, T.; Badie, J.; Flamant, G. Carbon nanotubes: Dynamics of synthesis processes. Carbon 2002, 40, 1621-1634. [CrossRef]

(C) 2016 by the authors; licensee MDPI, Basel, Switzerland. This article is an open access article distributed under the terms and conditions of the Creative Commons Attribution (CC-BY) license (http://creativecommons.org/licenses/by/4.0/). 